\title{
LA GESTIÓN DEL CONOCIMIENTO EN LAS ORGANIZACIONES Y LAS REGIONES: UNA REVISIÓN DE LA LITERATURA
}

THE KNOWLEDGE MANAGEMENT IN THE ORGANIZATIONS AND THE REGIONS: A REVIEW OF THE LITERATURE

\section{GESTÃO DO CONHECIMENTO NAS ORGANIZAÇÕES E REGIÕES: UMA REVISÃO DA LITERATURA}

\section{CALVO GIRALDO_Omaira}

Magíster en Estudios Interdisciplinarios del Desarrollo, Universidad del Cauca. Magíster en Emprendimiento y Desarrollo Empresarial, Universidad de Girona. Investigadora de la Universidad del Cauca. Email:ocalvo@unicauca.edu.co.

Recibido: 26 de junio de 2017 Aprobación definitiva:23 de mayo de 2018

DOI: http://dx.doi.org/10.22267/rtend.181901.91

\section{RESUMEN}

Este documento, presenta una revisión de la literatura, tomando como eje central la gestión del conocimiento y su desarrollo a nivel empresarial y regional. Esta investigación documental se ha realizado en el marco del proyecto "Red de Formación de Talento Humano para la Innovación Social y Productiva" con el objetivo de ser un referente para la formulación de una maestría en Gestión de Conocimiento y la Innovación en la Universidad del Cauca. En la primera parte se encuentra la conceptualización, los principales enfoques y autores más representativos. La segunda parte presenta la aplicación de la gestión del conocimiento en organizaciones basadas en conocimiento y las principales temáticas asociadas a este campo. Por último, está la gestión de conocimiento en el ámbito regional. Aquí se presentan el 
rol del conocimiento en el desarrollo regional, los principales modelos de innovación regional y los modelos de la gestión del conocimiento para la gestión en las regiones.

Palabras clave: Gestión del conocimiento, regiones, organizaciones de conocimiento

JEL: D83, O32, R11

\section{ABSTRACT}

This document presents a literature review, taking as the central focus the knowledge management and its development at the business and the regional level. This documentary research was conducted under the project "Human Resources Training Network for Social and Productive Innovation in the department of Cauca" with the aim of becoming a reference for the formulation of a master's degree in Knowledge Management and Innovation at the University of Cauca. In the first part there is the conceptualization, the main approaches and the most representative authors. The second part presents the application of knowledge management in knowledge-based organizations and major topics associated with this field. Finally, there is the knowledge management at the regional level. Here the role of knowledge in regional development, major regional innovation models and models of knowledge management for management in the regions are presented.

Keywords: Knowledge management, regions, knowledge organization JEL: D83, O32, R11

\section{RESUMO}

Este documento apresenta uma revisão de literatura, com foco principal em gestão do conhecimento e o seu desenvolvimento a nível empresarial e regional. Esta pesquisa documental foi conduzida com o projeto "Rede de Formação de Recursos Humanos para Inovação Social e Produtiva" com o objetivo de ser uma referência para a formulação de um mestrado em Gestão do Conhecimento e Inovação na Universidade de Cauca. Na primeira parte se encontra a concei- 
tualização, os enfoques principais e os autores mais representativos. A segunda parte consiste na aplicação de gestão do conhecimento em organizações baseadas em conhecimento e os principais tópicos associados com essa área. Por último é apresentada a gestão de conhecimento em nível regional. Aqui apresenta-se o papel do conhecimento no desenvolvimento regional, os principais modelos de inovação regional e os modelos da gestão do conhecimento para a gestão nas regiões.

Palavras-chave: gestão do conhecimento, regiões, organizações do conhecimento.

JEL: D83, O32, R11

\section{INTRODUCCIÓN}

En la economía del conocimiento, se considera que éste es un factor clave para la innovación y la competitividad de las organizaciones. Por tanto, surge la necesidad de buscar mecanismos para su creación, transformación y transferencia. Estos procesos hacen parte de la denominada gestión del conocimiento. Enfoque que ha sido ampliamente estudiado en el campo investigativo y que es uno de los principales paradigmas de la gestión empresarial.

Así mismo, se ha empezado a estudiar la incidencia que tiene la gestión de conocimiento en el ámbito regional, a través de la interacción de los diferentes actores, públicos y privados, de un territorio.

En tal sentido, este artículo proporciona una revisión de la literatura para resaltar la importancia de la gestión del conocimiento tanto en el ámbito organizacional como regional. La revisión de la literatura incluyó una búsqueda de bases de datos electrónicas y revistas en línea. La búsqueda se hizo por tema, es decir que los resultados arrojaron aquellos documentos que contuvieran la búsqueda en el título, el resumen o las palabras clave del artículo. El filtro se realizó de acuerdo con las categorías de las bases de datos, las áreas de investigación y títulos de fuentes. Todas estas categorías, relacionadas con las áreas de gestión de negocios y planeación del desarrollo. 


\section{RESULTADOS Y DISCUSIÓN}

\subsection{Gestión de Conocimiento}

La gestión de conocimiento puede ser definida como un proceso de transformación de la información y los activos intelectuales en valor perdurable (Tasmin and Yap 2010). Algunos autores la definen como la "habilidad de una empresa para incrementar el conocimiento tácito y crear las condiciones previas para el intercambio de información entre los empleados dentro de una unidad organizativa, $y$ entre las unidades de la organización" (Adams and Lamon 2003). Para otros autores, es "un proceso organizado, de creación, captura, almacenamiento, diseminación y uso del conocimiento dentro y entre organizaciones para mantener la ventaja competitiva" (Darroch 2003; Davenport and Prusak 2000).

La Gestión de Conocimiento se desarrolla en el marco de la llamada "nueva economía" o "Economía del conocimiento", en la que académicos y empresarios reconocen la importancia del conocimiento para lograr y mantener la ventaja competitiva de las organizaciones.

En la literatura se reconocen dos tipos de conocimiento: el conocimiento tácito y el conocimiento explícito (Nermien, 2003:84). El conocimiento tácito, es el conocimiento personal y usualmente depende del contexto. Incluye experiencias, intuiciones, ideas, visiones, habilidades y valores, por lo tanto es difícil de articular y transferir a otros (Krstić \& Petrović, 2011:95). Por el contrario el conocimiento explicito, es aquel que puede ser expresado y codificado fácilmente.

Autores como Metaxiotis, Ergazakis \& Psarras (2005), distinguen tres generaciones de la gestión de conocimiento. La primera está comprendida entre 1990 y 1995, conocida como la primera generación de la Gestión del Conocimiento. Durante este periodo, muchas iniciativas estuvieron focalizadas sobre la definición de Gestión de Conocimiento, investigando los beneficios potenciales en los negocios y diseñando proyectos específicos de gestión de conocimiento. 
La segunda generación surge a partir de 1996. En esta generación surgen puestos de trabajo dirigidos a Especialistas en Gestión de Conocimiento y se da un cambio en el discurso cotidiano de la gestión de las organizaciones. Finalmente, a partir de 2002 se habla de una tercera generación de la gestión del conocimiento, autores como Wiig (2002:5) manifiestan que:

Una diferencia con respecto a las generaciones anteriores es el grado en el que la tercera generación se integra con la filosofía, la estrategia, los objetivos, prácticas, sistemas y procedimientos de la empresa y la forma en que se convierte en parte de la vida diaria de cada empleado.

Desde la perspectiva de este autor, la gestión del conocimiento pasa a ser un elemento transversal y medular que permea todas las áreas de la organización.

\subsubsection{Modelos de Gestión de Conocimiento}

En la literatura, existen diferentes definiciones y modelos de Gestión de Conocimiento. A continuación se presenta la taxonomía de los diferentes modelos de gestión de conocimiento realizada por Kakabadse, Kakabadse \& Kouzmi (2003):

Modelo Cognitivo de la Gestión de Conocimiento: Hace referencia a los procesos de crear, localizar, capturar y compartir conocimientos y experiencias que puedan ser utilizados en la resolución de problemas. Entre los modelos más destacados se encuentra el "Modelo de Creación de Conocimiento", propuesto por Nonaka y Takeuchi (1995), que distinguen dos tipos de conocimiento, el tácito y el explícito. Los procesos que incluye el modelo son: socialización, externalización, combinación, internalización.

La socialización consiste en generar conocimiento tácito a partir de compartir experiencias, modelos mentales y creencias. La externalización es el proceso de convertir el conocimiento tácito en conocimiento explicito, es decir que este se pueda codificar para compartirlo con otros. El proceso de combinación consiste en crear conocimiento explícito a partir de conocimiento explícito que esté dentro o fuera de 
la organización. Por último, el proceso de internalización consiste en compartir en la organización el conocimiento explícito creado y convertirlo en conocimiento tácito.

Modelo en Red de la Gestión de Conocimiento: Este modelo surge en paralelo con las teorías de la organización en red y se centra en la adquisición, intercambio y transferencia de conocimiento (Kakabadse et al., 2003:83). Desde esta perspectiva son importantes las redes en las que están inmersas las personas, ya que esto amplía la posibilidad de adquirir conocimiento, que posteriormente se pueda compartir dentro de la organización.

Enfoque de Comunidad de Práctica de la Gestión de Conocimiento: Este es un modelo basado en la interacción que existe entre los diferentes niveles de la organización, donde los integrantes de la comunidad comparten recursos, valores y mutuamente buscan resolver problemas Kakabadse, Kakabadse y Kouzmin (2003:84)

\subsection{Gestión de Conocimiento en Organizaciones Basadas en Conocimiento}

Como se mencionó en el anterior apartado, los cambios en la economía han llevado a las organizaciones a ver el conocimiento como su principal activo y factor de competitividad. En el marco de esta premisa, la literatura revela que para algunas organizaciones el conocimiento es su elemento diferenciador, por tanto, emergen frases como: Organizaciones Intensivas en Conocimiento, Firmas Intensivas en Conocimiento y Organizaciones basadas en Conocimiento (Makani and Marche 2010) que se utilizan indistintamente.

Para Diana León (2012) las organizaciones basadas en conocimiento son "una estructura en la que compartir conocimiento es esencial para mantener la eficacia mientras los problemas son resueltos por un tipo especial de empleados llamados trabajadores del conocimiento". En este tipo de organizaciones, hay dos elementos importantes. En primer lugar, el Talento Humano es un factor determinante, al que se le atribuyen los éxitos o fracasos de la organización (Starbuck 1992) siendo aquellos trabajadores que están calificados y altamente capacitados, la mayor 
fuerza laboral. En segundo lugar, está la innovación, como factor inherente a la estrategia de estas organizaciones (Ditillo 2004; Ichijo and Nonaka 2006).

Algunos estudios empíricos han identificado los factores determinantes para llevar a cabo la gestión de conocimiento en organizaciones basadas en conocimiento. En primer lugar, está la Cultura del conocimiento, que es la que permite que haya expectativas comunes, experiencias compartidas y normas sociales que den forma a las actitudes y comportamientos (Tasmin 2008). Otro factor, son las Tecnologías de la Información. Al respecto (AngKim and Lee 2004) mencionan que con el fin de construir las capacidades de intercambio de conocimientos, la organización debe desarrollar una infraestructura integral que facilita los diversos tipos de conocimiento y comunicación. Finalmente, están las personas, se dice que son verdaderos agentes de negocios donde todos los bienes tangibles e intangibles son resultado de la acción humana y dependen en última instancia de las personas para su existencia continuada (Omar Sharifuddin Syed-lkhsan and Rowland 2004).

\subsection{Gestión de Conocimiento en el Ámbito Regional}

En el ámbito regional, el establecimiento de ventajas competitivas, está relacionado con la capacidad de crear conocimiento, transformarlo y utilizarlo (Harmaakorpi and Melkas 2005; Zhao and de Pablos 2011). Para Salonius y Kapyla (2013:584), la Gestión de Conocimiento Regional, es definida como "un proceso de creación de conocimiento donde los actores regionales participan en la formación e implementación de una visión y estrategias regionales para promover el desarrollo regional, aportando su capital intelectual al desarrollo de la red".

Dada la importancia que tienen las regiones en la creación de ventajas competitivas, existen varios modelos de innovación regional, que de acuerdo con Moulaert \& Sekia (2003), citado en (Naghizadeh, Elahi, Manteghi, Ghazinoory, \& Ranga, 2014:2495), comienzan a ser explorados en Europa y Estados Unidos, después de la segunda guerra mundial, como una respuesta a la necesidad de reconstrucción y la inversión en desarrollo industrial de las regiones. 
Algunos de los modelos que se encuentran en la literatura son: Distritos Industriales (Bagnasco 1997), Clúster Industrial (Porter 1990), Clúster Regional (Saxenian 1994), Sistema Regional de Innovación (Cooke 2002) (Doloreux and Parto 2005), Redes de Innovación (Rycroft 2003). La figura 1 muestra la evolución en el tiempo de los diferentes modelos.

Cada modelo tiene sus particularidades. Los distritos industriales, por ejemplo, para Beccattini (1989) citado en Galleto (2014:29), son "Una entidad socio territorial que se caracteriza por la presencia activa, de una comunidad de personas y de una población de empresas en un área natural e históricamente delimitada". Este modelo tiene sus raíces en los enfoques de equilibrio parcial y sistemas de fabricación flexibles de Marshall.

\begin{tabular}{|c|c|c|c|c|c|c|}
\hline \multicolumn{7}{|c|}{\begin{tabular}{|cc} 
Nuevos Espacios Industriales & $\begin{array}{c}\text { Difusión de Conocimiento } \\
\text { Clúster de Industrias basadas en Conocimiento }\end{array}$ \\
$\begin{array}{cc}\text { Sistemas de Producción Local } & \text { Clúster Regional }\end{array}$ \\
Distrito Industrial & Clúster Industrial
\end{tabular}} \\
\hline 1950 & 1960 & 1970 & 1980 & 1990 & 2000 & 2010 \\
\hline & & & & Entornos & $\begin{array}{r}\text { novación } \\
\text { ación Reg } \\
\text { Región de } \\
\text { Re }\end{array}$ & $\begin{array}{l}\text { ndizaje } \\
\text { Innovación }\end{array}$ \\
\hline
\end{tabular}

\section{Figura 1}

Evolución en el tiempo de los modelos de innovación regionales.

Fuente: Adaptado de (Naghizadeh et al. 2014)using a combination of quantitative (co-word analysis

El clúster regional, se basa en un estudio realizado en Silicon Valey (Saxenian 1994), en este se señala la importancia de las instituciones regionales y la cultura para el desarrollo. Modelos como los Sistemas Regionales de Innovación, las Redes de Innovación, y las Regiones de Aprendizaje, se dice que son una visión institucionalista del desarrollo económico (Martinelli, Moulaert, and Oana 2002). En estos modelos, aspectos como la teoría de redes, el capital social, el aprendizaje y las relaciones entre los actores, son muy importantes. 
Los Sistemas Regionales de Innovación, parten de la definición de Sistema Nacional de Innovación. Se dice que Philip Cooke (1992), fue el primer autor que utilizó este concepto definiéndolo como "subsistemas de generación y explotación de conocimiento que interactúan y se encuentran vinculados a otros sistemas regionales, nacionales $y$ globales, para la comercialización de nuevo conocimiento". En este modelo, la innovación es un proceso creativo que surge de la interacción entre diferentes actores.

Respecto al Modelo de Redes de Innovación (Belussi and Arcangeli 1998) plantean que la organización en red es un acuerdo institucional básico para hacer frente a la innovación sistémica. Hace énfasis en la importancia de las relaciones de cooperación entre las empresas como un mecanismo de articulación clave de configuraciones de red.

\subsubsection{Gestión de Conocimiento en Redes de Innovación}

Esta propuesta es realizada por (Harmaakorpi and Melkas 2005), quienes mencionan que los factores de competitividad están fuertemente relacionados con la habilidad de las regiones para crear y transformar el conocimiento en un entorno cambiante. En las redes de innovación son importantes las relaciones que existen al interior de ellas por tanto, temas como la generación de confianza y el capital social son imprescindibles. Al respecto Lundvall \& Borra (1999:30), plantean que es necesario "desarrollar un lenguaje y modos de interpretación común y sobre todo confianza con el fin de superar la incertidumbre que caracteriza los procesos de innovación".

Para alcanzar estos objetivos no solo se requiere de la proximidad y comunicación entre los actores. También es necesario que el conocimiento sea un recurso clave, para crear una base sólida (Nonaka and Reinmoller 1998). Por tanto, se requiere de la gestión de conocimiento, para crear, transformar y compartir conocimiento al interior de la red.

Para ello (Harmaakorpi and Melkas 2005), proponen un modelo (ver figura 2), basado en el modelo de conversión de conocimiento de (Nonaka and Takeuchi 1995), el cual consiste en cuatro fases: 
- Socialización: De conocimiento tácito a conocimiento tácito

- Externalización: De conocimiento tácito a conocimiento explícito

- Combinación: De conocimiento explícito a conocimiento explícito

- Internalización: De conocimiento explícito a conocimiento tácito

También, utiliza el concepto japonés de ba que fue introducido por (Nonaka and Konno 1998) a la teoría organizacional. El concepto parte de la idea de que el conocimiento necesita un contexto para ser creado, ya sea social, cultural o histórico, por tanto $\mathrm{Ba}$, es el contexto común en el que se comparte, crea y utiliza el conocimiento (Nonaka, Toyama, \& Konno, 2000:25). Cada fase del modelo de conversión de conocimiento necesita un ba específico:

- Socialización: Originating ba

- Externalización: Interacting ba

- Combinación: Cyber ba

- Internalization: Exercising ba

Por último el modelo incluye el concepto de conocimiento autotrascendente que fue introducido por (Scharmer 2001), definiéndolo como una habilidad para ver lo que otros no ven. Es decir la capacidad de ver el potencial que hay en el entorno y comprender las tendencias ocultas. Para Scharmer la gestión de conocimiento gira en torno a la interacción de las tres formas de conocimiento: tácito, explícito y autotrascendente.

A continuación se describe cada uno de los procesos:

- Visualización/Imagination ba: Tiene que ver con los estudios de futuro, utilizando técnicas como las previsiones, los escenarios y el panel de expertos. 


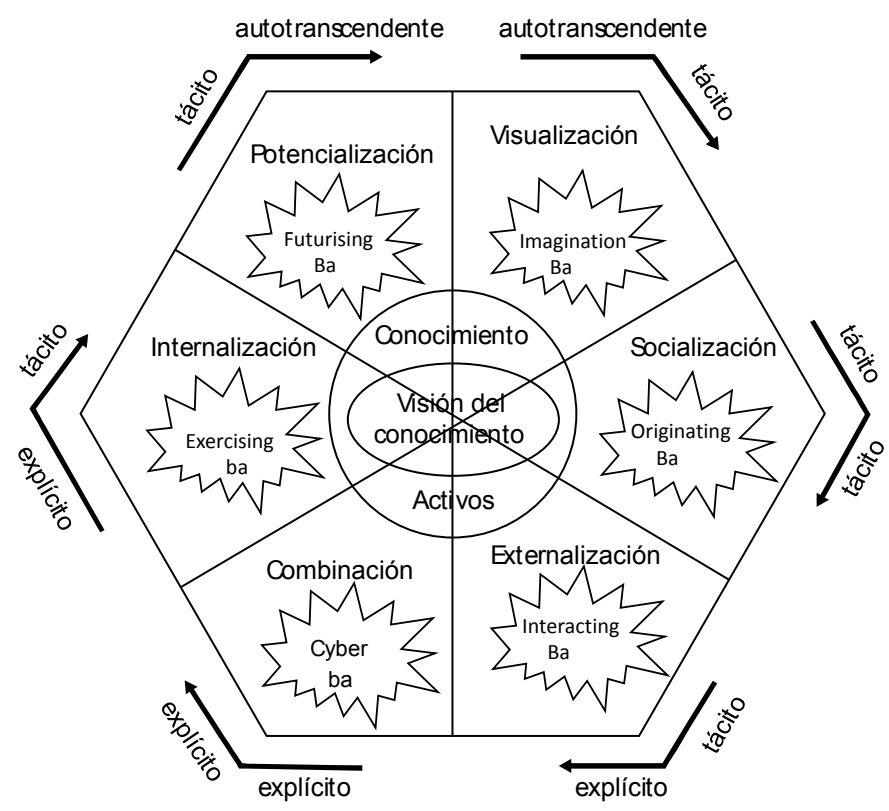

\section{Figura 2}

Gestión de Conocimiento en Redes de Innovación

Fuente: Adaptado de (Harmaakorpi and Melkas 2005)

- Socialización/Originating ba: El objetivo de este proceso es la generación de confianza entre los diferentes actores. Para ello, se organizan actividades en la que los diferentes actores tengan la oportunidad de compartir sus experiencias, lo que permite crear cohesión social.

- Externalización/Interacting ba: El objetivo de este proceso es promover el aprendizaje. Para lo cual, la organización de seminarios temáticos y de encuentros en los que se promueva la creatividad, hacen parte de las acciones que se desarrollan en esta fase.

- Combinación/Cyber ba: Se refiere a la utilización de recursos virtuales como las plataformas, a través de las cuales se comparte conocimiento dentro y fuera de la red.

- Internalización/Exercising ba: Permite que el conocimiento explícito de nuevo vuelva a ser tácito. Se hace uso de los procesos de mentoria en los que los actores más experimentados transfieren sus conocimientos. 
- Potencialización/Futurising ba: La creación de conocimiento al no tener un orden cronológico, permite que en esta etapa al igual que en la primera se prevea el futuro, haciendo uso de herramientas como el método Delphi.

\subsubsection{Transferencia de Conocimiento en Sistemas Regionales de Innovación}

Según (Jie and Zhengang 2006), en los sistemas regionales de innovación, desde la perspectiva del conocimiento, es crítico hacer que el conocimiento tácito sea fácil de transferir. Los autores proponen el uso de las tecnologías de la información y la comunicación (TIC's), aplicadas al modelo de creación de conocimiento (SECl) (Nonaka 1995) y su integración con elementos que faciliten la transferencia de conocimiento. (ver figura 3). A continuación se explican cada uno de los procesos propuestos en el modelo:

- Aplicación de las TIC's a los procesos de socialización: Propone la creación de espacios online, en los que trabajadores, investigadores y expertos puedan interactuar para discutir los temas de región. Esto permite crear un lenguaje común y capital social. 


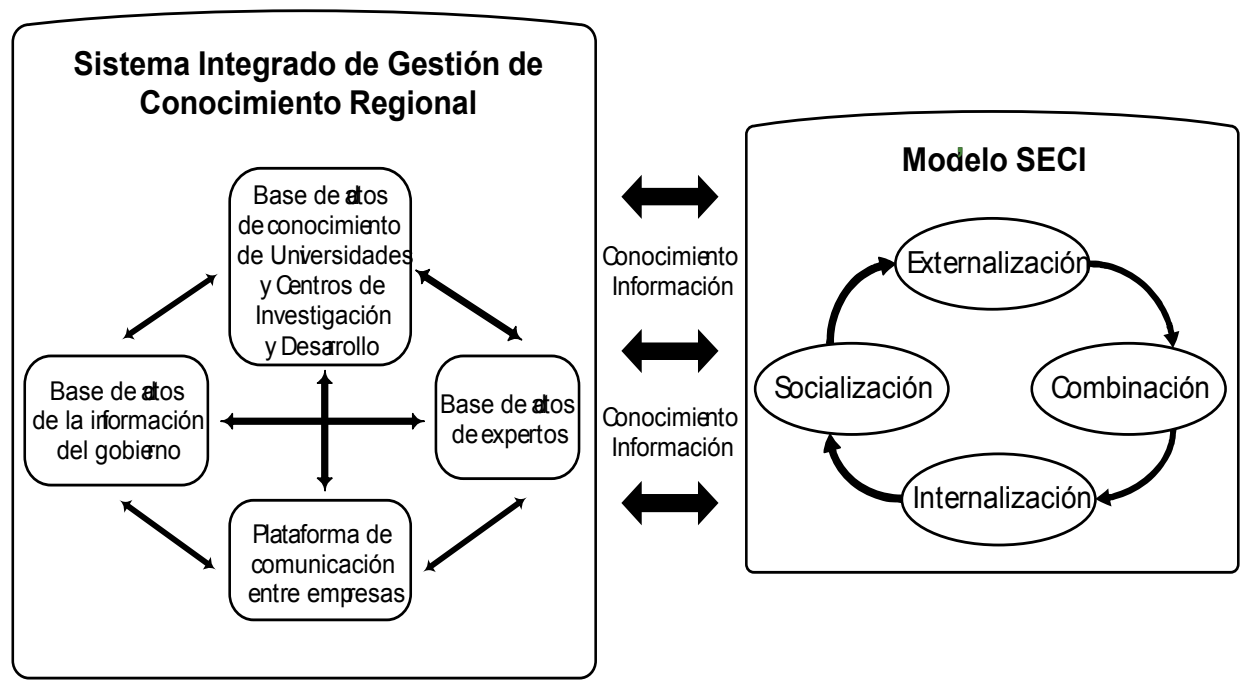

\section{Figura 3}

Integración del sistema de gestión de conocimiento al ámbito regional Fuente: Adaptado de (Jie and Zhengang 2006)

- Aplicación de las TIC's a los procesos de externalización: se puede brindar a los empleados un espacio virtual, en el que puedan colocar información útil, casos útiles y recordar sus experiencias. Esta información se comparte, se analiza y se codifica.

- Aplicación de las TIC's a los procesos de combinación: El conocimiento generado en la etapa de externalización, podrá ser clasificado e integrado, entonces el nuevo conocimiento podría añadirse al conocimiento existente para ampliar la base de conocimiento de la organización. Este conocimiento puede documentarse en formato digital y utilizar software inteligente para analizar y clasificar el conocimiento explícito.

- Aplicación de las TIC's a los procesos de internalización: A través del proceso de internalización los empleados transforman el conocimiento explícito en conocimiento tácito. El conocimiento explicito es adquirido de bases de datos de conocimiento que deben estar bien codificadas para facilitar el acceso y uso del conocimiento. 
Adicionalmente el modelo plantea un Sistema Integrado de Gestión de Conocimiento, con cuatro bases de datos que facilitan el uso e intercambio de conocimiento: Base de datos de la información del gobierno, base de datos de conocimiento de Universidades y Centros de Investigación y Desarrollo, base de datos de expertos y plataforma de comunicación entre empresas.

\subsubsection{Modelo de Gestión de Conocimiento para la Relación Universidad-Empresa-Estado}

Este modelo es diseñado por autores colombianos (Gutiérrez Rincón, Rincón Bergman, and Pérez Castano 2010), quienes en torno a las dinámicas del Sistema Regional de Ciencia, Tecnología e Innovación del Valle del Cauca, proponen la gestión del conocimiento como motor de la innovación y la transformación productiva en la región (ver figura 4).

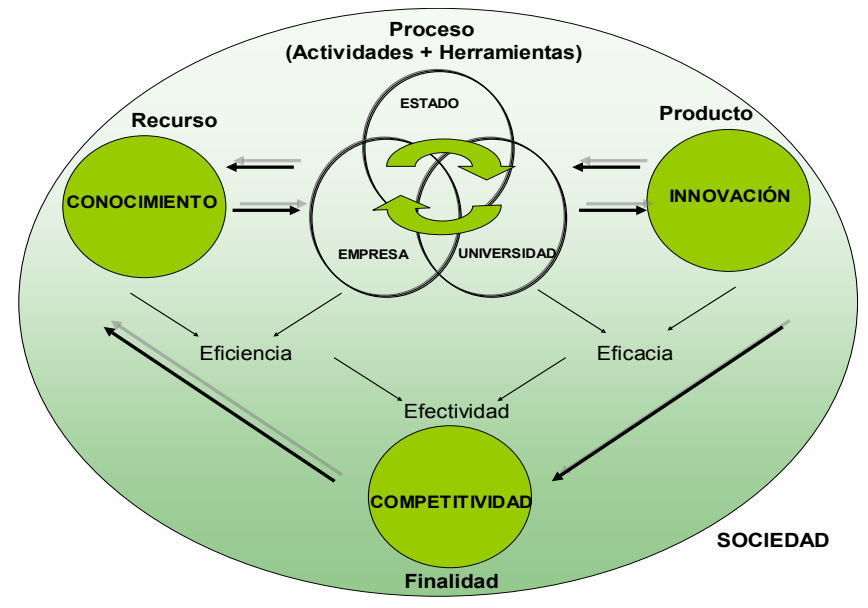

\section{Figura 4}

Modelo de Gestión del Conocimiento para la relación Universidad-Empresa-Estado.

Fuente:(Gutiérrez Rincón et al. 2010)

El modeloestá soportado porcuatro componentes: actores, actividades, herramientas de gestión de conocimiento y sistema de evaluación, cuyo objetivo es determinar la capacidad científica, tecnológica e innovadora de la región. Está basado en la interacción entre el modelo de Triple Hélice 
de la Innovación (Etzkowitz and Leydesdorff 2000) y las actividades de conocimiento propuestas por Holsapple y Joshi (2002) en el modelo participativo de la gestión de conocimiento.

El modelo de Triple Hélice, consiste en la generación de una infraestructura de conocimiento a partir de la interrelación entre Universidad-Empresa-Estado. Bajo este modelo se desarrollan iniciativas y alianzas estratégicas, entre las empresas, el gobierno y los grupos de investigación, para el desarrollo económico.

Por su parte, el modelo de Holsapple y Joshi se compone de cuatro actividades: adquisición, selección, interiorización y uso del conocimiento.

\subsubsection{Gestión de Conocimiento Regional desde la Teoría de la Gestión}

La propuesta de este modelo la realizan Zhao y de Pablos (2011:40). Estos autores mencionan que la gestión de conocimiento es el resultado de combinar las ciencias de la gestión y las ciencias de la información. Para ellos, el objetivo de la gestión de conocimiento regional es:

El establecimiento de la innovación regional a través de ambientes de aprendizaje, sistemas de capacitación y educación a nivel regional y políticas que conduzcan a mejorar la calidad de la fuerza laboral. Además, cultivar las redes regionales de innovación y el capital social de la región, de tal manera que se propicie la cooperación de los modos de aprendizaje, tales como el aprendizaje individual, aprendizaje organizacional y el aprendizaje institucional.

La gestión, en términos generales incluye las funciones de planeación, organización, liderazgo y control (Ver figura 5). A partir de estas funciones los autores realizan un análisis de la gestión de conocimiento regional Así: 


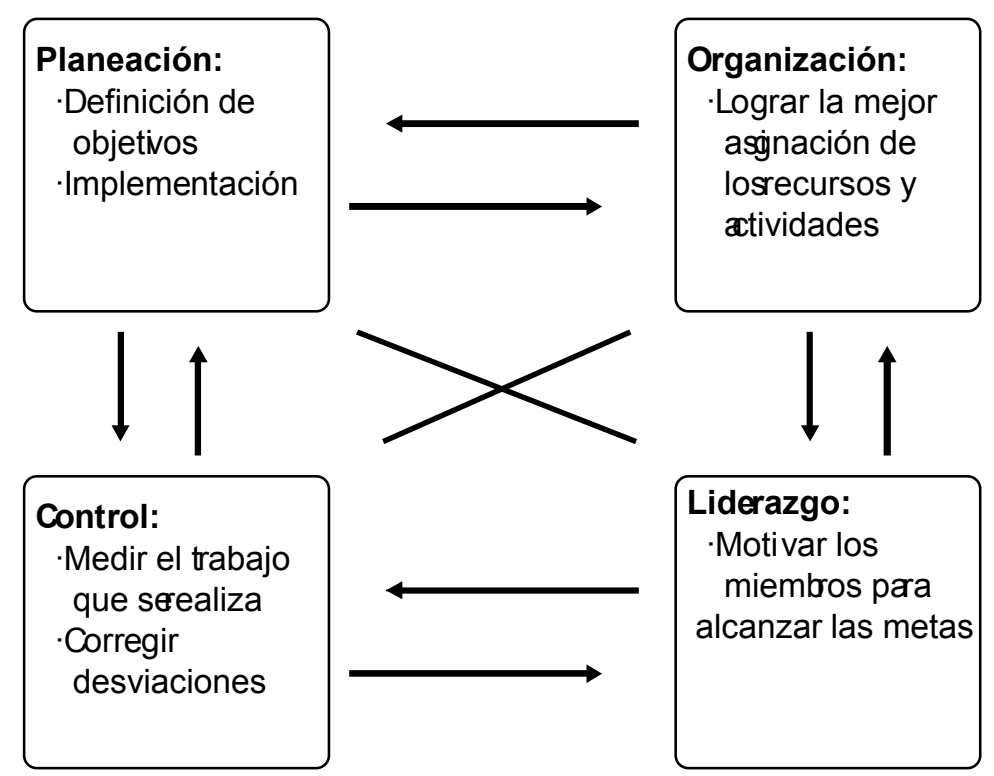

Figura 5.

Funciones de la gestión

Fuente: Adaptado de (Zhao and de Pablos 2011)

Planeación: Esta función se realiza a través de la implementación de políticas regionales de ciencia y tecnología e incluye programas: de investigación científica, de revolución tecnológica, de prospectiva tecnológica, de investigación y desarrollo, y de cooperación científica y tecnológica.

Organización: La gestión del conocimiento regional, concibe esta función a través del Sistema Regional de Innovación, que es en sí una forma de organización eficaz compuesta por empresas, instituciones científicas y tecnológicas, universidades e instituciones intermediarias donde hay unos roles claramente definidos. Para el caso de las empresas, estas son consideradas el pilar fundamental de la innovación; por su parte las Universidades e instituciones son la fuente de creación de conocimiento; las instituciones intermediarias permiten articular los diferentes actores; y el gobierno actúa como facilitador de las actividades regionales de innovación. 
Control: Esta función consiste en resolver problemas regionales y garantizar la equidad en el intercambio de conocimiento. Estos problemas pueden darse en la producción, el intercambio y la circulación de conocimiento.

Liderazgo: Esta función corresponde al gobierno, que debe encargarse de proveer un ambiente de aprendizaje e innovación a través de condiciones sistemáticas, soportadas en políticas. Estas políticas pueden ser: de incentivos, de orientación y de coordinación.

\subsubsection{Gestión de Conocimiento en Redes de Desarrollo Regional, a partir del Modelo de Creación de Conocimiento y el Capital Intelectual}

En este modelo los autores proponen combinar dos marcos de trabajo: la gestión de conocimiento y el capital intelectual (Salonius and Kapyla 2013) more appropriate investments (e.g. for ICT infrastructure (ver figura 6).

La propuesta plantea que el modelo SECI (Nonaka and Takeuchi 1995), tiene sus bases en la conversión de conocimiento a través de cuatro fases: Socialización, Externalización, Combinación, Internalización. 


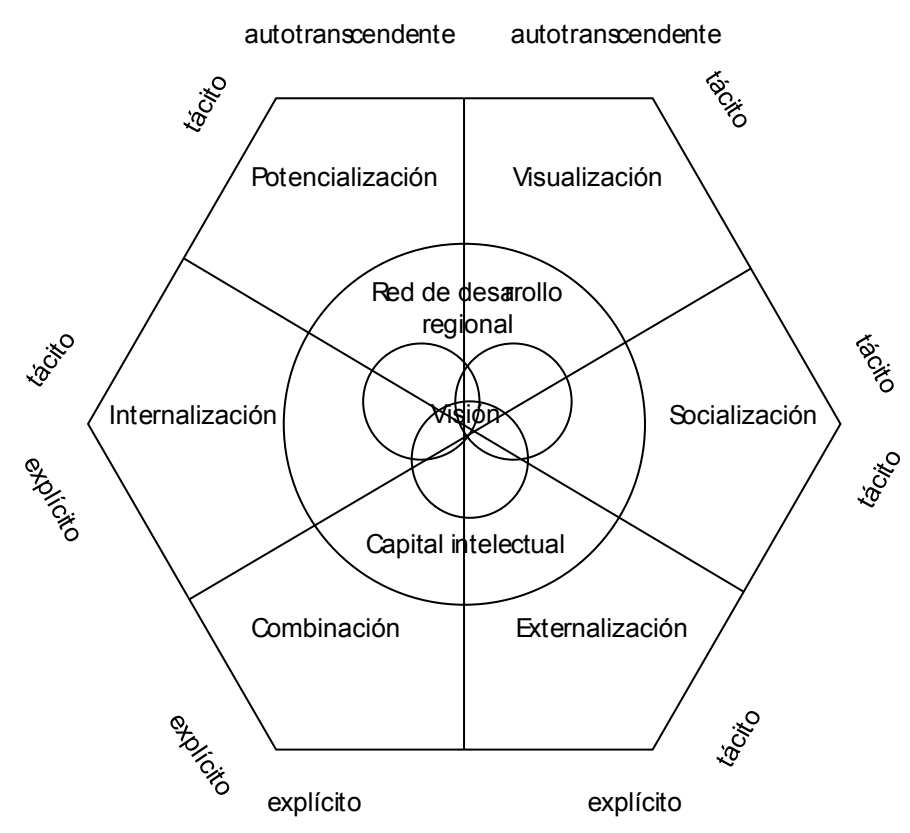

\section{Figura 6}

Combinación del Modelo SECI con el Capital Intelectual Fuente: Adaptado de (Salonius and Kapyla 2013)more appropriate investments (e.g. for ICT infrastructure

Desde esta perspectiva, la conversión de conocimiento, facilita los procesos de aprendizaje colectivo, incrementando así el conocimiento de la red.

La incorporación del Capital Intelectual se hace para incrementar los beneficios de la creación de conocimiento a través de los activos intelectuales, los cuales son indispensables para crear valor en la empresa. Estos se clasifican en cuatro tipos (Nonaka et al. 2000):

1. Los activos de conocimientos experimentales, son el conocimiento tácito que se comparte, a través de experiencias comunes, entre los miembros de la organización y con los clientes, proveedores y socios. 
2. Los activos de conocimiento conceptual, que significa conocimiento explícito articulado a través de imágenes, símbolos y lenguaje, que permiten crear valor para los clientes.

3. Los activos de conocimiento sistémicos, se refieren al conocimiento explícito sistematizado y empaquetado que es fácilmente transferible.

4. Los activos de conocimiento de rutina, es el conocimiento tácito rutinario, incorporado en las acciones y la práctica.

Los autores sostienen que el enfoque del Capital Intelectual, ofrece una perspectiva más amplia y práctica sobre estos activos. Este concepto está clasificado en tres categorías, capital humano, capital estructural y capital relacional. El capital humano se refiere al conocimiento tácito y explícito que poseen los individuos, incluye las actitudes, aptitudes y valores (Martin-de Castro, Lopez-Saez, and Delgado-Verde 2011). El capital estructural tiene que ver con el capital tecnológico y organizacional que posee la organización, incluye temas como la cultura, estructura y aprendizaje organizacional (Martin-de Castro et al. 2011). El capital relacional hace referencia al valor que tiene para la organización la relación con sus stakeholders ${ }^{1}$ (Clientes, proveedores, socios)(Martin-de Castro et al. 2011). Como una subcategoría dentro del modelo de capital intelectual, los autores agregan el capital social, en el que la comunicación y la confianza son aspectos claves para una red de desarrollo regional.

Los modelos presentados muestran la capacidad de las regiones para soportar procesos de innovación y generar ventajas competitivas a partir de la gestión del conocimiento. Así mismo, muestran que este es un proceso interactivo que requiere de la participación de los diferentes actores institucionales y de la definición de roles para cada uno de ellos.

1 Grupos y/o individuos que puedan afectar o que son afectados por el logro de los objetivos de la organización 


\section{CONCLUSIONES.}

Tanto en los Sistemas Regionales de Innovación, las Regiones de Aprendizaje y las Organizaciones Basadas en Conocimiento, el conocimiento tácito cobra un papel fundamental, por lo tanto la gestión del Talento Humano y el desarrollo de competencias blandas son fundamentales.

En el nivel regional, el conocimiento debe ser gestionado a partir de la interacción entre los actores Universidad-Empresa-Estado, quienes deben tener unos roles claramente definidos y en los que exista un nodo articulador.

Los modelos de innovación regional pueden ser un referente para la toma de decisiones en la administración pública, acerca de las acciones que se deben llevar a cabo para el desarrollo de un territorio a través del conocimiento y la innovación.

Existen diferentes enfoques de la gestión de conocimiento, por un lado está el tecnológico, en el que a través de las Tecnologías de la Información y la Comunicación se accede a la información y se procesa. Por otro lado, está el enfoque orientado a los procesos organizacionales y las personas en el que se destacan temas como el desarrollo organizacional y los activos intelectuales.

Tanto a nivel empresarial como regional, la gestión de conocimiento se inclina por aspectos como la interacción humana, el desarrollo del diálogo a nivel formal e informal y la creación de redes entre las personas y organizaciones.

\section{Agradecimientos}

La autora expresa sus agradecimientos al grupo de investigación Modelos Regionales de Competitividad y al proyecto Red de Formación de Talento Humano para la Innovación Social y Productiva en el Departamento del Cauca, financiado por el Sistema General de Regalías y ejecutado por la Universidad del Cauca. 


\section{REFERENCIAS}

(1) Adams, Garry L. and Bruce T. Lamon. (2003). "Knowledge Management Systems and Developing Sustainable Competitive Advantage." Journal of Knowledge Management 7(2):142-54.

(2) AngKim, Soonhee and Hyangsoo Lee. (2004). "Organizational Factors Affecting Knowledge Sharing Capabilities in E-Government: An Empirical Study."

(3) Bagnasco, Arnaldo. (1997). "Tre Italie: La Problematica Territoriale Dello Sviluppo Economico Italiano." II Mulino 255.

(4) Belussi, Fiorenza. and Fabio. Arcangeli. (1998). "A Typology of Flexible and Evolutionary Firms." Research Policy 27:415-28.

(5) Cooke, Philip. (2002). "Knowledge Economies Clusters Learning and Cooperative Advantage." Annals of the Association of American Geographers 93(4):944-46.

(6) Cooke, Philip. (1992). "Regional Innovation Systems: Competitive Regulation in the New Europe." Geoforum 23:365-82.

(7) Darroch, Jenny. (2003). "Developing a Measure of Knowledge Management Behaviors and Practices." Journal of Knowledge Management 7(5):41-54.

(8) Davenport, Thomas and Laurence. Prusak. (2000). "Working Knowledge: How Organizations Manage What They Know." Harvard Business School Press.

(9) Ditillo, Angelo. (2004). "Dealing with Uncertainty in Knowledge-Intensive Firms: The Role of Management Control Systems as Knowledge Integration Mechanisms." Accounting, Organizations and Society 29(3-4):401-21.

(10) Doloreux, David and Saeed Parto. (2005). "Regional Innovation Systems: Current Discourse and Unresolved Issues." 27(2):133-53.

(11) Etzkowitz, Henry. and Loet. Leydesdorff. (2000). "The Dynamics of Innovation: From National Systems and 'Mode 2' to a Triple Helix of University-IndustryGovernment Relations." Research Policy 29(2):109-23.

(12) Galletto, Vittorio. (2014). "Distritos Industriales E Innovación." 
(13) GutierrezRincon, Viviana, Gladys RinconBergman, and Bernardo Perez Castano. (2010). "Knowledge Management as a Tool for the Productive Transformation of a Region in a Developing Country." Pp. 426-35 in PROCEEDINGS OF THE 11TH EUROPEAN CONFERENCE ON KNOWLEDGE MANAGEMENT, edited by $\mathrm{E}$ Tome.

(14) Harmaakorpi, Vesa and Helina Melkas. (2005). "Knowledge Management in Regional Innovation Networks: The Case of Lahti, Finland." EUROPEAN PLANNING STUDIES 13(5):641-59.

(15) Holsapple, Clyde and Kshiti Joshi.( 2002). "Knowledge Management: A Threefold Framework." The Information Society 18(1):47-64.

(16) Ichijo, Kazuo and Ikujiro Nonaka. (2006). "Knowledge Creation and ManageMent: New Challenges for Managers." Oxford University Press.

(17) Jie, Xue and Zhang Zhengang. (2006). "The Research on the Application Strategies of Information and Communication Technologies to Promote the Knowledge Transfer in Regional Innovation System." Pp. 138-45 in APSCC: 2006 IEEE ASIA-PACIFIC CONFERENCE ON SERVICES COMPUTING, PROCEEDINGS.

(18) Kakabadse, Nada K., Andrew Kakabadse, and Alexander Kouzmin. (2003). "Reviewing the Knowledge Management Literature: Towards a Taxonomy." Journal of Knowledge Management 7(4):75-91.

(19) Krstić, Bojan and Bojan Petrović. (2011). "THE ROLE OF KNOWLEDGE MANAGEMENT IN DEVELOPING CAPABILITIES FOR INCREASING ENTERPRISE' S Bojan Krsti Ć , Bojan Petrovi Ć." 8(1991):275-86.

(20) Leon, Ramona-Diana.(2012). "Strategic Factors for Developing Sustainable Knowledge Based Organizations." Pp. 618-25 in PROCEEDINGS OF THE 13TH EUROPEAN CONFERENCE ON KNOWLEDGE MANAGEMENT, VOLS 1 AND 2, edited by JG Cegarra.

(21) Lundvall, Bengt Åke and Susana Borrás. (1997). "The Globalising Learning Economy: Implications for Innovation Policy." $T$ Bruxelles, European Commission. Targeted Socio-Economic Research.

(22) Makani, Joyline and Sunny Marche. (2010). "Towards a Typology of KnowledgeIntensive Organizations: Determinant Factors." KNOWLEDGE MANAGEMENT RESEARCH \& PRACTICE 8(3):265-77. 
(23) Martin-de Castro, Gregorio, Pedro Lopez-Saez, and Miriam Delgado-Verde. (2011). "Towards a Knowledge-Based View of Firm Innovation. Theory and Empirical Research." JOURNAL OF KNOWLEDGE MANAGEMENT 15(6):87174.

(24) Martinelli, Flavia, Frank Moulaert, and Ailenei Oana. (2002). "Territorial Innovation Models: A Critical Survey of The International Literature." Pp. 289302 in Social Innovation. Governance and Communty Building. Lille.

(25) Metaxiotis, Kostas, Kostas Ergazakis, and John Psarras. (2005). "Exploring the World of Knowledge Management: Agreements and Disagreements in the Academic/practitioner Community." Journal of Knowledge Management 9(2):618.

(26) Moulaert, Frank. and Farid. Sekia. (2003). "Territorial Innovation Models: A Critical Survey." Regional Studies 37(3):289-302.

(27) Naghizadeh, Reza, Shaban Elahi, Manoochehr Manteghi, Sepehr Ghazinoory, and Marina Ranga.(2014). "Through the Magnifying Glass: An Analysis of Regional Innovation Models Based on Co-Word and Meta-Synthesis Methods." Quality and Quantity 49(6):2481-2505. Retrieved ("http://dx.doi.org/10.1007/ s11135-014-0123-7).

(28) Nermien, Al-Ali. (2003). Comprehensive Intellectual Capital Management Stepby-Step. John Wiley.

(29) Nonaka, Ikujiro and Hirotaka. Takeuchi. (1995). The Knowledge-Creating Company: How Japanese Companies Create the Dynamics of Innovation.

(30) Nonaka, Ikujiro. and Noboru. Konno. (1998). "The Concept of 'ba': Building a Foundation for Knowledge Creation." Management Review 40(3):40-54.

(31) Nonaka, Ikujiro. and Patrick. Reinmoller. (1998). "The Legacy of Learning: Toward Endogenous Knowledge Creation for Asian Economic Development." WZB Jahrbuch 401-33.

(32) Nonaka, Ikujiro., Rryoko. Toyama, and Noboru Konno. (2000). "SECl, Ba and Leadership: A Unified Model of Dynamic Knowledge Creation." Long Range Planning 33(1):5-34.

(33) Omar Sharifuddin Syed-Ikhsan, Syed and Fytton. Rowland. (2004). "Knowledge Management in A Public Organization: A Study On The Relationship Between Organizational Elements and The Performance of Knowledge Transfer." Journal Of Knowledge Management 8(2):95-111. 
(34) Porter, Michael E. (1990). "The Competitive Advantage of Nations." Free Press.

(35) Rycroft, Robert. (2003). "Technology-Based Globalization Indicators: The Centrality of Innovation Network Data." Technology in Society 25(3):299-317.

(36) Salonius, Henna and Jonna Kapyla. (2013). "Exploring the Requirements of Regional Knowledge-Based Management." JOURNAL OF KNOWLEDGE MANAGEMENT 17(4):583-97.

(37) Saxenian, AnnaLee. (1994). Regional Advantage: Culture and Competition in Silicon Valley and Route 128. edited by Harvard University Press.

(38) Scharmer, Claus Otto. (2001). "Self-Transcending Knowledge: Sensing and Organizing around Emerging Opportunities." Journal of Knowledge Management 5(2):137-51.

(39) Starbuck, William H. (1992). "Learning by Knowledge-Intensive Firms." Journal of Management Studies 29(6):713-40.

(40) Swart, Juani and Nicholas Kinnie.( 2003). "Sharing Knowledge in KnowledgeIntensive Firms." Human Resource Management Journal 13(2):60-75.

(41) Tasmin, Rosmaini. (2008). "Knowledge Management and Innovation Among Manufacturing Firms in Peninsular Malaysia." Unpublished PhD Thesis.

(42) Tasmin, Rosmaini and L. S. Yap. (2010). "Determining Factors of Knowledge Management Implementation in Knowledge-Based Organizations." Pp. 49-54 in PROCEEDINGS OF KNOWLEDGE MANAGEMENT 5TH INTERNATIONAL CONFERENCE 2010, edited by MA Baharom, F and Mahmuddin, M and Yusof, $Y$ and Ishak, WHW and Saip. Retrieved (http://eprints.uthm.edu.my/1836/1/ DETERMINING_FACTORS_OF_KNOWLEDGE_MANAGEMENT_DR_ ROSMAINI_TASMIN.pdf).

(43) Wiig, Karl M. (2002). "New Generation Knowledge Management : What May We Expect ?" People-Focused Knowledge Management 1-9.

(44) Zhao, Jingyuan and F de Pablos. (2011). "Regional Knowledge Management: The Perspective of Management Theory." BEHAVIOUR \& INFORMATION TECHNOLOGY 30(1, SI):39-49. 